\title{
1,5-Diaminonaphtalene is a Highly Performing Electron-Transfer Secondary-Reaction Matrix for Laser Desorption Ionization Mass Spectrometry of Indolenine-Based Croconaines
}

\author{
Cosima D. Calvano, ${ }^{\dagger, \S}$ Maria Annunziata M. Capozzi, ${ }^{\ddagger \odot}$ Angela Punzi, ${ }^{\ddagger 0}$ Gianluca M. Farinola, ${ }^{\ddagger \odot}$
} Tommaso R. I. Cataldi, ${ }^{*},,+$ a and Francesco Palmisano ${ }^{\dagger, \ddagger}$

${ }^{\dagger}$ Centro di Ricerca Interdipartimentale SMART, ${ }^{*}$ Dipartimento di Chimica and ${ }^{\S}$ Dipartimento di Farmacia-Scienze del Farmaco, Università degli Studi di Bari Aldo Moro, Campus Universitario, Via E. Orabona, 4, 70126 Bari, Italy

Supporting Information

ABSTRACT: Croconaine dyes are appealing molecules synthesized via the condensation of croconic acid and reactive electrondonating aromatic or heterocyclic systems. Here, matrix-assisted laser desorption/ionization (MALDI) mass spectrometry (MS) investigation of indolenine-based croconaines is presented for the first time. Archetype proton-transfer matrices, such as 2,5dihydroxybenzoic acid (DHB) and $\alpha$-cyano-4-hydroxycinnamic acid (CHCA), 9-aminoacridine (9AA) as the protonating/ deprotonating matrix, and electron-transfer (ET) secondaryreaction matrices, such as 1,5-diaminonapthalene (DAN) and trans-2-[3-(4-t-butyl-phenyl)-2-methyl-2-propenylidene]malononitrile (DCTB), were investigated. DHB, CHCA, and 9AA generate a mix of odd-electron molecular ions and protonated,

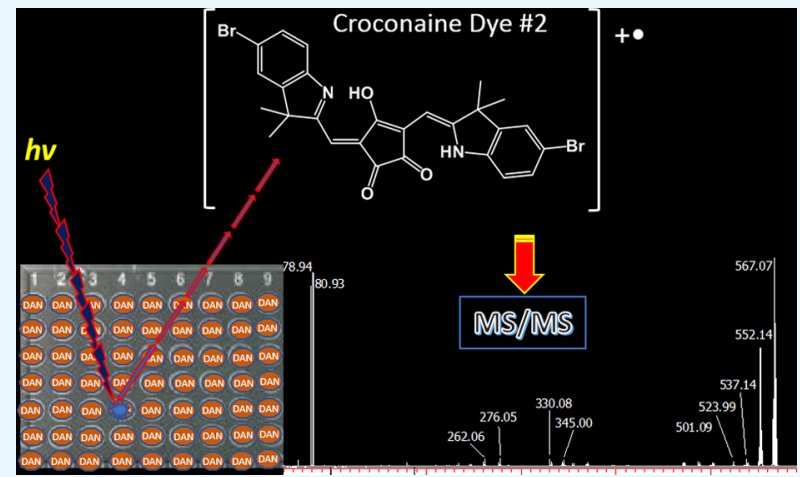
sodiated, and potassiated adducts. Among the ET matrices, DAN was found to be capable of directing the ionization process toward the exclusive formation of odd-electron molecular ions $\mathrm{M}^{+\bullet}$ without fragmentation. MALDI tandem MS provides useful structural characterization of croconaine dyes, thus making identification very straightforward for all investigated compounds. Interestingly the fragmentation of bromo-containing croconaines revealed, for the first time, the gas-phase formation of a bromime cation $[\mathrm{Br}]^{+}$.

\section{INTRODUCTION}

Croconaines are donor-acceptor-donor type zwitterionic compounds synthesized from croconic acid (4,5-dihydroxy-4cyclopentene-1,2,3-trione ${ }^{1}$ ) via a one-pot condensation reaction with electron-rich (hetero)aromatic compounds or heterocyclic methylene-active bases. $^{2-4}$ Croconaines exhibit narrow and intense absorption bands in the NIR, and their optical properties can be finely tuned either by changing the donor portions, conjugated to the croconic acid core, ${ }^{5,6}$ or by inserting diverse substituents on the donor moieties. ${ }^{3,7}$ Very recently, synthetic strategies leading to derivatives with tailored photochemical properties have been reviewed. ${ }^{8}$

Despite the low solubility of croconaines in both organic and aqueous media, additional features, such as high photothermal conversion efficiency, ${ }^{9}$ excellent chemical and photothermal stability, ${ }^{10}$ and strong solvatochromism, ${ }^{11}$ have attracted considerable interest toward their application as effective NIR-absorbing dyes. Furthermore, a recent study has provided evidences that croconaine dyes could represent promising candidates for cancer photothermal therapy. ${ }^{12}$ Interest in these dyes, however, is not restricted to the above-mentioned fields; indeed acido- and ionochromisms are revealed to be useful for their applications in optical sensing of cations ${ }^{13,14}$ or anions, ${ }^{15}$ whereas their third-order nonlinear optical properties appear potentially useful for ultrafast optical devices. ${ }^{16}$ Recently, a small library of indolenine-based croconaines differing for the substituents on the donor moieties has been synthesized and their molecular structure fully investigated (by density functional theory (DFT) calculations, NMR spectroscopy, and X-ray diffraction) together with their solid-state organization. ${ }^{17}$ Ambipolar semiconducting properties have been obtained in thin-film transistors, demonstrating indoleninebased croconaines as a new class of molecular materials for organic electronics. ${ }^{17}$

Chemical and structural characterization of croconaines is typically performed by ${ }^{1} \mathrm{H}$ - and ${ }^{13} \mathrm{C}$ NMR, IR spectroscopy, and X-ray diffraction. At present, mass spectrometry (MS)based techniques appear still confined to a marginal role in characterizing croconaines. Indeed, electron or chemical ionization methods typically employed in gas chromatography (GC)-MS are useless since indolenine-based croconaines cannot be investigated by GC due to molecular masses well

Received: September 28, 2018

Accepted: December 6, 2018

Published: December 19, 2018 
Table 1. Summary of All Croconaines (1-6) Investigated by MALDI MS ${ }^{a}$

\begin{tabular}{|c|c|c|c|c|c|c|}
\hline Chemical structure of croconaine dyes & \# & Substituents & $\begin{array}{l}\text { Chemical } \\
\text { formula }\end{array}$ & $\begin{array}{c}\text { Monoisotopic } \\
\text { mass (Da) }\end{array}$ & LUMO $(e V)^{a}$ & HOMO $(\mathrm{eV})^{\mathrm{a}}$ \\
\hline & 1 & $\begin{array}{l}R_{1}, R_{3}, R_{4}=H \\
R_{2}=P h-O-P h\end{array}$ & $\mathrm{C}_{51} \mathrm{H}_{40} \mathrm{~N}_{2} \mathrm{O}_{5}$ & 760.2937 & -3.98 & -5.26 \\
\hline & 2 & $\begin{array}{l}\mathrm{R}_{1}, \mathrm{R}_{3}, \mathrm{R}_{4}=\mathrm{H} \\
\mathrm{R}_{2}=\mathrm{Br}\end{array}$ & $\mathrm{C}_{27} \mathrm{H}_{22} \mathrm{Br}_{2} \mathrm{~N}_{2} \mathrm{O}_{3}$ & 579.9997 & -4.03 & -5.33 \\
\hline & 3 & $\begin{array}{l}\mathrm{R}_{1}, \mathrm{R}_{3}, \mathrm{R}_{4}=\mathrm{H} \\
\mathrm{R}_{2}=\mathrm{F}\end{array}$ & $\mathrm{C}_{27} \mathrm{H}_{22} \mathrm{~F}_{2} \mathrm{~N}_{2} \mathrm{O}_{3}$ & 460.1598 & -3.96 & -5.28 \\
\hline & 4 & $\begin{array}{l}\mathrm{R}_{1}, \mathrm{R}_{2}, \mathrm{R}_{3}=\mathrm{H} \\
\mathrm{R}_{4}=\mathrm{F}\end{array}$ & $\mathrm{C}_{27} \mathrm{H}_{22} \mathrm{~F}_{2} \mathrm{~N}_{2} \mathrm{O}_{3}$ & 460.1598 & -4.03 & -5.34 \\
\hline & 5 & $\begin{array}{l}R_{1}, R_{3}=H \\
R_{2}, R_{4}=F\end{array}$ & & 496.1410 & -4.09 & -5.38 \\
\hline & 6 & $\begin{array}{l}\mathrm{R}_{1}, \mathrm{R}_{3}, \mathrm{R}_{4}=\mathrm{H} \\
\mathrm{R}_{2}=\mathrm{Ph}-\mathrm{NO}_{2}\end{array}$ & $\mathrm{C}_{39} \mathrm{H}_{30} \mathrm{~N}_{4} \mathrm{O}_{7}$ & 666.2114 & -4.05 & -5.32 \\
\hline
\end{tabular}

${ }^{a}$ Energy levels of HOMO and LUMO were determined by cyclic voltammetry in $\mathrm{CH}_{2} \mathrm{Cl}_{2}$ solution. For experimental details, see ref 17 .

above $400 \mathrm{Da}$. So far, electrospray ionization mass spectrometry (ESI-MS) has been only used to assess the molecular mass of the synthesized croconaines (typically detected as deprotonated molecules), whereas structural characterization studies by tandem or multistage MS are lacking. ${ }^{13,17,18}$ Note that the above-mentioned poor solubility of croconaines could hamper ESI-MS measurement since the best suited solvent ensuring adequate solubility could not be fully compatible with the ESI source. To the best of our knowledge, matrix-assisted laser desorption/ionization (MALDI) $\mathrm{MS}^{19}$ has never been exploited for croconaine dyes. Here, the selection of an appropriate matrix capable of ensuring efficient desorption/ ionization of the analytes with minimal or null fragmentation still represents an open issue. ${ }^{20}$ Considering the croconaine structure, containing basic and acidic residues on an extensively conjugated electron system, common and wellknown proton-transfer (PT) and/or electron-transfer (ET) matrices are likely candidates; ${ }^{21-23}$ note that PT and ET are potentially competing processes and that the prevalence of one over the other depends on the analyte-matrix combination. A set of five representative matrices (exhibiting different proton affinities and ionization potentials (IPs), vide infra) was selected which included the following: (i) archetype PT matrices such as 2,5-dihydroxybenzoic acid (DHB) and $\alpha$ cyano-4-hydroxycinnamic acid (CHCA); (ii) 9-aminoacridine (9AA) as a protonating/deprotonating matrix; ${ }^{24}$ (iii) 1,5 diaminonapthalene (DAN) capable of acting as the PT/ET matrix, ${ }^{21,22}$ and (iv) trans-2-[3-(4-t-butyl-phenyl)-2-methyl-2propenylidene]malononitrile (DCTB), an effective and wellrecognized nonprotic ET matrix. ${ }^{25}$ Performance of these matrices is presented, compared, and discussed in the following using, as model compounds, five previously described ${ }^{19}$ indolenine-based croconaines and a newly synthesized one (see later). Among the investigated matrices, DAN revealed a soft (i.e., absence of analyte fragmentation) and a highperformance ET matrix leading solely to the formation of an odd-electron molecular ion. The fragmentation patterns observed in MALDI MS/MS experiments are also discussed; the fragmentation of bromo-containing croconaines revealed the formation of a bromine cation $\left([\mathrm{Br}]^{+}\right)$in the gas phase, which has never been reported before in MALDI MS experiments.

\section{RESULTS AND DISCUSSION}

2.1. Laser Desorption Ionization (LDI)-MS experiments. Indolenine-based croconaines investigated here are reported in Table 1 along with their chemical formula, substituent groups on each indolenine moiety, and monoisotopic mass; note that all croconaines are symmetrical and differ by the donor substituents, namely, heteroatoms as fluorine (\#3-5) and bromine (\#2) or aromatic moieties as 4phenoxy-phenyl (\#1) and 4-nitro-phenyl (\#6). Highestoccupied and lowest-unoccupied Kohn-Sham orbital (HOMO and LUMO) energy levels, previously estimated from cyclic voltammetry (CV) experiments, ${ }^{17}$ are also quoted, given their importance in rationalizing the laser desorption ionization mechanism (vide infra). Unfortunately, DFT calculations of HOMO and LUMO energy levels, which generally provide ionization potential (IP) and electron affinity (EA) values, respectively, in good agreement with the experimental data in a vacuum, ${ }^{26}$ are not available for the investigated compounds. It follows that HOMO and LUMO energy levels obtained by CV provide only rough (but still useful) estimates of IP and EA values mainly because the HOMO/LUMO energies are scaled in vacuum, whereas the reduction/oxidation potentials are measured in solution. ${ }^{26,27}$ As expected for analytes with extended aromatic $\pi$-electron systems, all investigated croconaines are characterized by particularly low IPs (around $5.3 \mathrm{eV}$ ), suggesting a facile direct photoionization process in the gas phase upon irradiation by $\mathrm{Nd}$ :YLF laser (photon energy, $3.6 \mathrm{eV}$ ). Indeed, the LDI mass spectrum of, e.g., croconaine \#5 (see Figure S1, Supporting Information) shows the distinct formation of a $[\mathrm{M}]^{+\bullet}$ radical ion at $\mathrm{m} / \mathrm{z} 496$ that can be easily rationalized invoking a twophoton ionization process. ${ }^{28}$ The absolute signal intensity is not particularly high, which is consistent with a broad and poor absorption band at the laser wavelength of $345 \mathrm{~nm}$, too far from the absorption maximum at ca. $780 \mathrm{~nm}$ (Figure S2, Supporting Information). A similar behavior is shared by all other investigated compounds. The above LDI findings suggest that direct analyte photoionization should be likely expected also in MALDI experiments; however, due to the excess of matrix, such a process cannot play a significant role since secondary ionization of an analyte by a matrix is far more likely than the direct ionization of analyte, unless very high analyte/ matrix ratios are used, ${ }^{29}$ which is not the case.

2.2. Matrix Evaluation. Figure 1 displays the positive-ion MALDI MS spectra of croconaine \#1 using CHCA (a), DHB (b), and 9AA (c) as matrices. The base peak at $m / z 761.302$ in the MALDI spectrum obtained using CHCA as the matrix (plot (a)) was assigned to the protonated adduct $[\mathrm{M}+\mathrm{H}]^{+}$. Interestingly, the concomitant occurrence of an odd-electron molecular ion, $\mathbf{M}^{+}$, at $m / z 760.298$, with a remarkably high yield (ca. 70\%, see the inset of plot (a)) can be observed; since 


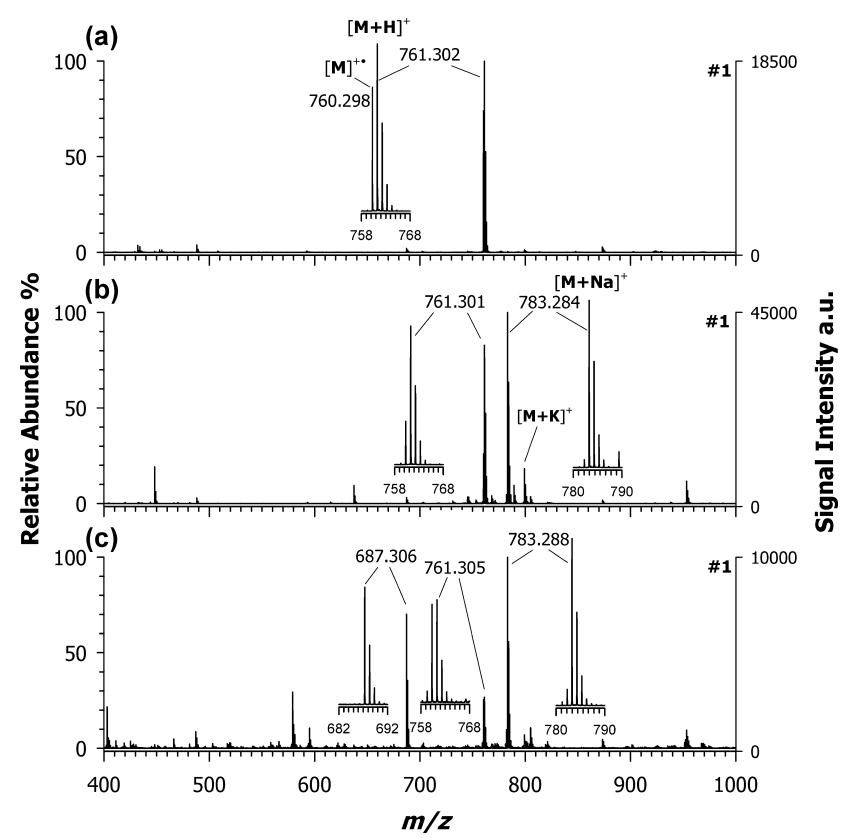

Figure 1. Positive-ion mass spectra by MALDI-time-of-flight (ToF) of croconaine dye \#1 $\left(\mathrm{M}, \mathrm{C}_{51} \mathrm{H}_{40} \mathrm{~N}_{2} \mathrm{O}_{5}\right)$ obtained by using (a) CHCA, (b) DHB, and (c) 9AA as matrices. Enlarged isotopic patterns of the main peaks detected in each spectrum are shown in insets.

direct photoionization can provide only a marginal contribution (see the previous paragraph), the formation of $\mathrm{M}^{+\bullet}$ should be mainly due to secondary reactions, indicating that PT and ET are competing processes. Cationization by $\mathrm{Na}^{+}$and/or $\mathrm{K}^{+}$ adduction is completely absent in the case of CHCA. Different findings were experienced by using DHB (plot (b)) and 9AA (plot (c)) as matrices; the formation of sodiated adducts (see the base peak at $m / z 783$ ) is now the preferred ionization process. A small contribution of a $\mathrm{K}^{+}$adduct also appears, whereas the $\mathrm{M}^{+\bullet}$ relative abundance at $m / z 760.298$ is, in both cases, around $30 \%$ referred to the base peak at $\mathrm{m} / z 783$. Notably, the $[\mathrm{M}+\mathrm{Na}]^{+} /[\mathrm{M}+\mathrm{H}]^{+}$relative abundance ratio increases considerably passing from CHCA to DHB to 9AA. For a given analyte, cationization can become the preferred ionization route when matrices with increasing PA are used, since the analyte must compete with the neutral matrix for the available protons in plume secondary reactions. ${ }^{30}$ Indeed, PA values (see Table 2) increase in the order CHCA $<$ DHB < 9AA, thus providing a strong support to the above interpretation. As multiple ion formation for a given analyte is generally considered deleterious, especially for (semi)quantitative application of MALDI MS, ET matrices were tested to force the ionization process toward the formation of a single species, i.e., the radical cation.

Table 2. Thermodynamic Data of Matrices Used in This Work for Croconaine Examination by MALDI MS $^{a}$

$\begin{array}{lccclc} & \text { CHCA } & \text { DHB } & \text { 9AA } & \text { DCTB } & \text { DAN } \\ \text { PA }(\mathrm{kcal} / \mathrm{mol}) & 201.0^{b} & 204.4^{b} & 233.4^{b} & \text { n.a. } & 225.0^{b} \\ \text { EA }(\mathrm{kcal} / \mathrm{mol}) & 40.1^{d} & 17.7^{d} & 14.4^{d} & 53.3^{36} & -9.18^{c} \\ \mathrm{IP}(\mathrm{eV}) & 8.80^{d} & 8.20^{d} & 7.23^{d} & 8.54^{36} & 6.84^{c}\end{array}$

${ }^{a} \mathrm{PA}$ : proton affinity, EA: electron affinity, IP: ionization potential. ${ }^{b}$ Data obtained by refs $23-30$ and references herein. ${ }^{c}$ See ref 37. ${ }^{d}$ Data obtained by Mol-Instincts Chemical Property Database (https://www.molinstincts.com/home/index/).
Figure 2 shows the positive-ion MALDI mass spectra of croconaine \#1 using (a) DCTB and (b) DAN as representative

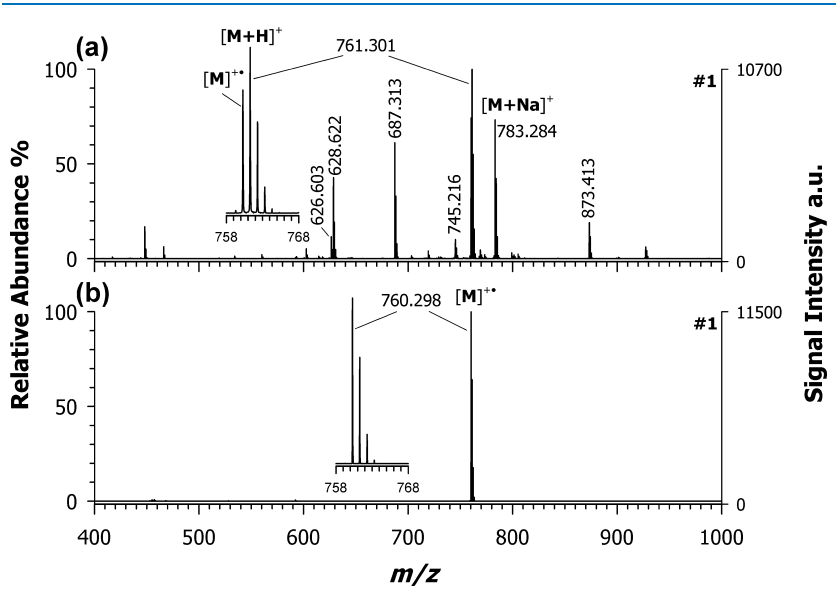

Figure 2. Positive-ion mass spectra by MALDI-ToF of croconaine dye \#1 obtained by using (a) DCTB and (b) DAN as ET matrices. Enlarged isotopic pattern in insets of the main peaks detected in each spectrum.

ET matrices. Unexpectedly, DCTB was unable to drive the ionization process toward the prevalent formation of the radical cation, the protonated adduct being the most abundant species, followed by the radical ion and the sodiated adduct at $m / z$ 783.284. Moreover, some matrix-related signals were recorded (at $m / z 628$ and 873 ) together with an analyte fragment ion at $m / z 687$ (observed also using 9AA as a matrix, see Figure 1c); such a fragment could be assigned as $\mathrm{C}_{45} \mathrm{H}_{34} \mathrm{~N}_{2} \mathrm{O}_{4} \mathrm{Na}^{+}$, likely originated from the neutral losses of phenol $\left(\mathrm{C}_{6} \mathrm{H}_{6} \mathrm{O}\right)$ and $\mathrm{H}_{2}$. Note that the DCTB spectrum in the negative-ion mode LDI MS shows the exclusive formation of the radical anion $\mathrm{M}^{-} \bullet$ (data not shown) whereas in positiveion mode, the radical cation $\mathrm{M}^{+\bullet}$ is formed together with a plethora of other species such as $[\mathrm{M}+\mathrm{H}]^{+},[\mathrm{M}+\mathrm{Na}]^{+},[\mathrm{M}+$ $\mathrm{K}]^{+}$, and $\left[\mathrm{M}-\mathrm{CH}_{3}\right]^{+}$, just to cite the most relevant ones. ${ }^{31}$ This could be the reason why DCTB failed to behave as a pure ET transfer matrix (in the positive mode) for croconaines; however, due to the lack of thermochemical data, the ionization mechanisms were not investigated further.

A different picture was obtained with DAN (plot (b) in Figure 2). Indeed, DAN can drive the ionization process toward the formation of the odd-electron molecular ion (see the base peak at $m / z 760.298$ ) with nearly $100 \%$ yield (see the inset of Figure $2 b$ ). Besides, compared with DCTB, no matrixrelated peaks occurred in either positive- or negative-ion modes (data not shown), thus indicating that DAN shows the best performance as the matrix (vide infra). From a thermodynamic point of view, ET secondary reactions are possible when the IP of the analyte is lower than that of the matrix $\left(\mathrm{IP}_{\mathrm{A}}<\mathrm{IP}_{\mathrm{M}}\right)$. Among the investigated matrices, DAN possesses the lowest IP value $6.84 \mathrm{eV}^{32}$ which is roughly 1.5 $\mathrm{eV}$ higher than that of cocronaines' counterpart; such a difference in IPs should, however, be enough for an effective ET with the additional advantage of a low excess energy deposited in the $\mathrm{M}^{+\bullet}$ ion that justifies the absence of analyte fragmentation.

To confirm these results, all other synthesized croconaines were investigated by using CHCA as the PT matrix and DAN as the ET matrix. As seen for croconaine dye \#1, the use of CHCA generated systematically two or more contributions 
(see Figure S3, Supporting Information), whereas DAN was confirmed to be the election matrix for this class of compounds (Figure S4, Supporting Information). Indeed, as reported in Figure 3, it was possible to detect the radical cation with nearly

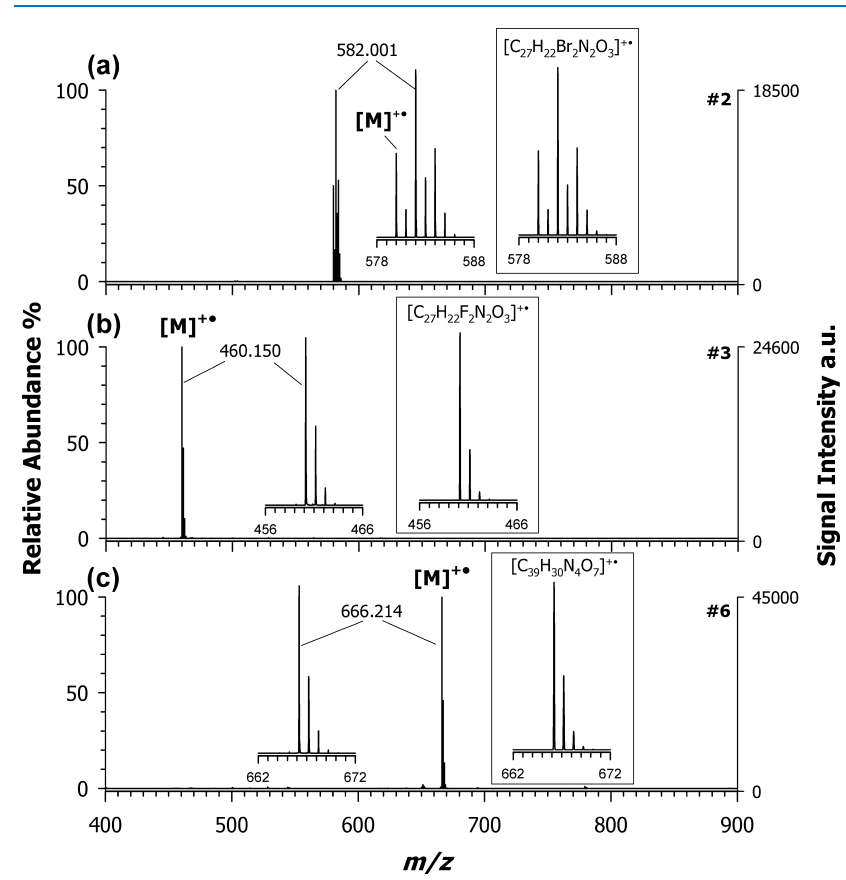

Figure 3. Positive-ion mass spectra by MALDI-ToF of (a) croconaine dyes \#2 ( $\left.\mathrm{M}, \mathrm{C}_{27} \mathrm{H}_{22} \mathrm{Br}_{2} \mathrm{~N}_{2} \mathrm{O}_{3}\right)$, (b) \#3 ( $\left.\mathrm{M}, \mathrm{C}_{27} \mathrm{H}_{22} \mathrm{~F}_{2} \mathrm{~N}_{2} \mathrm{O}_{3}\right)$, and (c) \#6 $\left(\mathrm{M}, \mathrm{C}_{39} \mathrm{H}_{30} \mathrm{~N}_{4} \mathrm{O}_{7}\right)$ obtained by using DAN as the matrix. Inset of plot (a) shows the simulated isotopic pattern of a radical molecular ion with composition $\left[\mathrm{C}_{27} \mathrm{H}_{22} \mathrm{Br}_{2} \mathrm{~N}_{2} \mathrm{O}_{3}\right]^{+}$, which is in good agreement with the experimental pattern of croconaine dyes \#2.

$100 \%$ yield, in the spectra of the croconaine dye \#2 at $\mathrm{m} / \mathrm{z}$ 582.001 (plot (3a)), dye \#3 at $m / z 460.150$ (plot (3b)), and dye \#6 at $\mathrm{m} / z 666.214$ (plot (3c)). All of these signals are in full agreement with theoretical isotope calculations obtained considering only the radical cation contribution.

2.3. MALDI MS/MS of Croconaine Dyes. After selecting the best matrix for croconaine dyes, the next step was structural characterization by tandem MS analyses. Figure 4

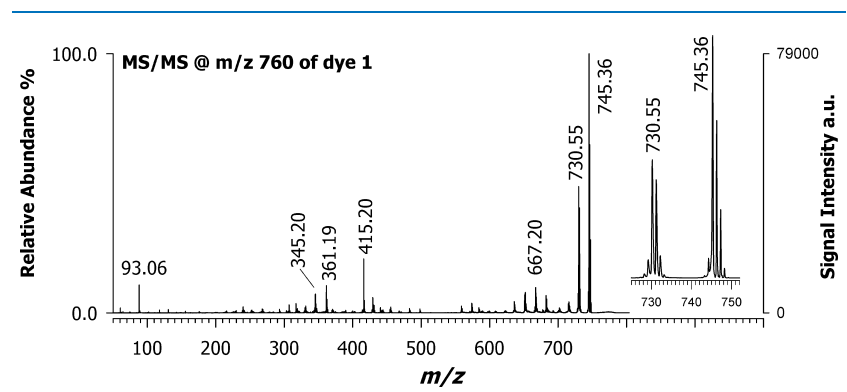

Figure 4. Positive MALDI-ToF/ToF mass spectrum of croconaine dye $\# 1$ precursor ion at $m / z 760$ using DAN as the matrix.

shows the positive-ion MALDI MS/MS spectrum of the precursor ion at $m / z 760$ referred to croconaine dye \#1, with an isolation window of $\pm 3 \mathrm{~m} / z$ units using DAN as the matrix. After isolating and fragmenting the molecular ion $\left([\mathrm{M}]^{+\bullet}\right)$, a main peak at $m / z 745.36$ was observed due to the facile loss of a methyl radical $\left({ }^{\bullet} \mathrm{CH}_{3}\right)$, most likely from the dihydropyrrole ring. The second abundant peak at $\mathrm{m} / z 730.55$ could be generated by the alternative loss of neutral formaldehyde $\left(\mathrm{CH}_{2} \mathrm{O}\right)$, in which the croconic acid core may be involved, or more likely from a consecutive loss of another methyl radical $\left({ }^{\bullet} \mathrm{CH}_{3}\right)$ from the second dihydropyrrole ring. The ion cluster centered at $m / z 667.20$ can be correlated to the radical loss of phenol $\left(\mathrm{C}_{6} \mathrm{H}_{5} \mathrm{O}^{\bullet}\right)$. For the less intense ions in the spectrum, several hypothetical product ions can be proposed. For instance, the peak observed at $\mathrm{m} / z 415.20$ can be explained by considering the breaking of the indolenine core. Also, product ions at $m / z 361.19$ and 345.20 are related to bond breaking in the gas phase on different points of the structure, which more likely involves the further loss of lateral substituents. The peak at $m / z 93.06$ is most likely due to the phenoxy ion arising from the side substituents.

Figure 5 shows the positive MALDI MS/MS spectrum of the croconaine dye \#2 (isolation window $\pm 5 \mathrm{~m} / \mathrm{z}$ units) using

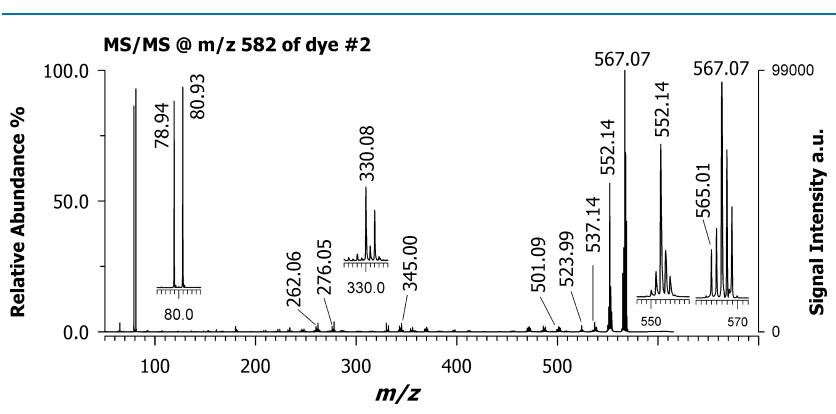

Figure 5. Positive MALDI-ToF/ToF mass spectrum of croconaine dye \#2 with the precursor ion at $m / z 582$ using DAN as the matrix. Note the occurrence of a cluster at $\mathrm{m} / z 78.94$ and 80.93 due to bromine cation.

DAN as the matrix. This compound contains two symmetrical substituents bearing bromide as a heteroatom. When fragmenting the ion cluster centered at $\mathrm{m} / z$ 582, two leading product ions were detected, most likely corresponding to the successive loss of two radical methyl moieties from the dihydropyrrole rings, as occurred with dye \#1 (see signals at $\mathrm{m} / z 567.07$ and 552.14); an additional methyl loss can generate the small peak at $\mathrm{m} / z 537.14$. The ion at $\mathrm{m} / z 330.08$ could originate from different losses as its isotopic pattern appears to be an envelope of at least two contributions. For instance, it could be due to the neutral loss of bromobenzene $\left(\mathrm{C}_{6} \mathrm{H}_{5} \mathrm{Br}\right)$ from one side and bromide $(\mathrm{Br})$ from the other substituent together with methyl loss or more likely it could be explained as the loss of the whole substituent 5-bromo-3,3dimethylindoline from one side and methyl from the other symmetric side. The peak at $\mathrm{m} / z 276$ is most likely generated from that at $m / z 332$ assigned as croconic moiety missing a methyl group upon loss of ethene-1,2-dione $\left[\mathrm{C}_{2} \mathrm{O}_{2}\right]$.

Besides other low-intensity product ions likely generated by successive losses of water $(18 \mathrm{Da})$ or methyl radical $(15 \mathrm{Da})$, an interesting ion doublet was detected at $\mathrm{m} / z 78.94$ and 80.93. According to both the accurate mass and isotopic pattern, this cluster can be easily attributed to bromine cation, $[\mathrm{Br}]^{+}$, formed via heterolytic cleavage. The presence of this ion species may appear anomalous since bromine follows the octet rule and usually is detected in a negative-ion mode as a bromide ion. Although much less intense, the product ions at $m / z 501-503$ are also evident following the initial loss of a bromine $\mathrm{Br}^{\bullet}$ radical (i.e., homolytic cleavage). The use of the same croconaine dye \#2 and a PT matrix such as CHCA 
(Figure S5, Supporting Information) provides no evidence of bromine cation. This is the first time that the $\mathrm{Br}^{+}$has been described in a MALDI experiment but it is not completely unusual using other techniques, including inductively coupled plasma-MS $^{33}$ and glow-discharge ionization source. ${ }^{34,35}$

\section{CONCLUSIONS}

Here, we have reported for the first time that DAN can be successfully used as a matrix to explore intact croconaine dyes by means of MALDI MS in a positive-ion mode. The prevailing ionization process of croconaines by DAN seems to be an ET secondary-reaction mechanism, thus hindering the splitting of ion contribution to protonated and cationized adducts, as observed with other examined matrices. Tandem MS of the radical molecular ions was found to be very useful to characterize the structure of these dyes. The most singular result was observed with the gas-phase formation of bromine cation on croconaines bearing $\mathrm{Br}$ as heteroatom substituents. This outcome has never been reported before, at least under MALDI MS experimental conditions. It is plausible that DAN will be actively used as a major MALDI matrix in identifying novel croconaine dyes.

\section{MATERIALS AND METHODS}

4.1. Chemicals. Water, acetone, methanol, chloroform, 1,5diaminonapthalene (DAN), trans-2-[3-(4-t-butyl-phenyl)-2methyl-2-propenylidene]malononitrile (DCTB), 2,5-dihydroxybenzoic acid (DHB), and $\alpha$-cyano-4-hydroxycinnamic acid (CHCA) were obtained from Sigma-Aldrich (Milan, Italy), whereas 9-aminoacridine hemihydrate was purchased from Acros Organics (Morris Plains, NJ). All solvents used were of LC-MS grade. A Mass Standard Kit for calibration was purchased from $\mathrm{AB}$ Sciex (Ontario, Canada).

4.2. Instrumentation. All experiments were performed using a 5800 MALDI-ToF/ToF analyzer (AB SCIEX, Darmstadt, Germany) equipped with a neodymium-doped yttrium lithium fluoride (Nd:YLF) laser (345 nm), in a reflectron positive mode, with a typical mass accuracy of 10 ppm. In MS and MS/MS modes, 1000 laser shots were typically accumulated by a random rastering pattern, at laser pulse rates of 400 and $1000 \mathrm{~Hz}$, respectively; each shown mass spectrum was averaged on at least five single mass spectra (1000 laser shots each). The laser fluences were within 1.9-2.6 $\mathrm{J} / \mathrm{m}^{2}$ depending on the matrix employed. MS/MS experiments were performed setting a potential difference of $1 \mathrm{kV}$ between the source and the collision cell; ambient air was used as the collision gas with a medium pressure of $10^{-6}$ Torr. The delayed extraction time was set at $280 \mathrm{~ns}$.

DataExplorer software 4.0 (AB Sciex) was used to control the acquisitions and to perform the initial elaboration of data, whereas SigmaPlot 11.0 was used to graph the final mass spectra. ChemDraw Pro 8.0.3 (CambridgeSoft Corporation, Cambridge, MA) was employed to draw chemical structures. Isotopic pattern contribution was generated by Xcalibur 4.0 with Foundation 3.1 (Thermo Fisher Scientific).

4.3. Croconaine Synthesis. Indolenine-based croconaines bearing different substituents on the donor moieties were synthetized following a protocol based on condensation of croconic acid (1 equiv) and of indolenine ( 2 equiv) in a refluxing mixture of toluene and butanol. Synthesis and characterization of croconaines \#1-4 and \#6 (see Table 1) were carried out in accordance with a previously reported procedure. ${ }^{17}$ Synthesis of the new croconaine \#5, namely, 3(5,7-difluoro-3,3-dimethyl-1,3-dihydro-indol-2-ylidenemethyl)5-(5,7-difluoro-3,3-dimethyl-3H-indol-2-ylmethylene)-4-hydroxy-cyclopent-3-ene-1,2-dione, is described in the Supporting Information.

4.4. Sample Preparation and MALDI MS Analysis. All investigated croconaines were dissolved in chloroform at a concentration of $2.0 \mathrm{mM}$. CHCA, DHB, and 9AA matrices were prepared in methanol at a concentration of $50 \mathrm{mM}$, whereas DAN and DCTB matrices were prepared in acetone at a concentration of $32 \mathrm{mM}$. Unless otherwise specified, $5 \mu \mathrm{L}$ of each croconaine solution was mixed (1:1, v/v ratio) with matrix solution and $1 \mu \mathrm{L}$ of mixture was spotted on the target plate, dried in the dark, and analyzed by MALDI MS.

WARNING: Caution should be reserved when handling DAN due to its possible carcinogenetic effects. Exposure to this compound should be minimized, and fume hoods should always be used.

\section{ASSOCIATED CONTENT}

\section{Supporting Information}

The Supporting Information is available free of charge on the ACS Publications website at DOI: 10.1021/acsomega.8b02575.

Positive-ion LDI-ToF mass spectrum of croconaine dye \#5 (Figure S1); absorption UV-visible-NIR spectrum of compound \#5 (Figure S2); positive-ion mass spectra by MALDI-ToF of croconaine dye \#2 obtained by using (A) CHCA, (B) DHB, and (C) 9AA as matrices (Figure S3); positive-ion mass spectra by MALDI-ToF of (a) croconaine dyes \#5 $\left(\mathrm{C}_{27} \mathrm{H}_{20} \mathrm{~F}_{4} \mathrm{~N}_{2} \mathrm{O}_{3}\right)$ and (b) \#4 $\left(\mathrm{C}_{27} \mathrm{H}_{22} \mathrm{~F}_{2} \mathrm{~N}_{2} \mathrm{O}_{3}\right)$ obtained by using DAN as a matrix (Figure S4); positive MALDI-ToF/ToF mass spectra of croconaine dye \#2 using CHCA as a matrix (Figure S5); synthesis of crocronaine \#5: ${ }^{1} \mathrm{H}$ NMR spectrum of croconaine \#1 (500 MHz, $\mathrm{CDCl}_{3}$ ) (Figure S6); ${ }^{1} \mathrm{H}$ NMR spectrum of croconaine \#2 (600 MHz, $\left.\mathrm{CDCl}_{3}\right)$ (Figure S7); ${ }^{1} \mathrm{H}$ NMR spectrum of croconaine \#3 (500 $\mathrm{MHz}, \mathrm{CDCl}_{3}$ ) (Figure S8); ${ }^{1} \mathrm{H}$ NMR spectrum of croconaine \#4 (500 MHz, $\left.\mathrm{CDCl}_{3}\right)$ (Figure S9); ${ }^{1} \mathrm{H}$ NMR spectrum of croconaine \#5 (500 $\left.\mathrm{MHz}, \mathrm{CDCl}_{3}\right)$ (Figure S10); ${ }^{13} \mathrm{C}$ NMR spectrum of croconaine \#5 $\left(125 \mathrm{MHz}, \mathrm{CDCl}_{3}\right.$ ) (Figure S11); ${ }^{1} \mathrm{H}$ NMR spectrum of croconaine \#6 (500 MHz, $\mathrm{CDCl}_{3}$ ) (Figure S12); cyclic voltammogram of croconaine \#5 (Figure S13) (PDF)

\section{AUTHOR INFORMATION}

\section{Corresponding Author}

*E-mail: tommaso.cataldi@uniba.it.

ORCID

Maria Annunziata M. Capozzi: 0000-0002-5661-3792

Angela Punzi: 0000-0001-5948-6373

Gianluca M. Farinola: 0000-0002-1601-2810

Tommaso R. I. Cataldi: 0000-0003-4811-8959

Notes

The authors declare no competing financial interest.

\section{ACKNOWLEDGMENTS}

This work was supported by the project PONa3_00395/1 "BIOSCIENZE \& SALUTE (B\&H)" of Italian Ministero per l'Istruzione, l'Università e la Ricerca (MIUR). 


\section{REFERENCES}

(1) Ito, M.; West, R. New Aromatic Anions. IV. Vibrational Spectra and Force Constants for C4O4-2 and C5O5-2. J. Am. Chem. Soc. 1963, 85, 2580-2584.

(2) Simard, T. P.; Yu, J. H.; Haley, N. F.; Detty, M. R. Soluble, infrared-absorbing croconate dyes from 2,6-di-tert-butyl-4- methylchalcogenopyrylium salts. J. Org. Chem. 2000, 65, 2236-2238.

(3) Avirah, R. R.; Jyothish, K.; Ramaiah, D. Infrared absorbing croconaine dyes: Synthesis and metal ion binding properties. J. Org. Chem. 2008, 73, 274-279.

(4) Kurdiukova, I. V.; Kulinich, A. V.; Ishchenko, A. A. Near-infrared squarate and croconate dianions derived from tetranitrofluorene. New J. Chem. 2012, 36, 1564-1567.

(5) Capozzi, M. A. M.; Punzi, A.; Babudri, F.; Musio, R.; Farinola, G. M. Synthesis and Computational Study of Semicroconaines and nonSymmetric Croconaines. J. Org. Chem. 2018, 83, 14396-14405.

(6) Keil, D.; Hartmann, H.; Reichardt, C. Synthesis and Spectroscopic Characterization of New NIR Absorbing (2-Thienyl)and (4-Dialkylaminoaryl)-substituted Croconic Acid Dyes. Liebigs Ann. Chem. 1993, 1993, 935-939.

(7) Puyol, M.; Encinas, C.; Rivera, L.; Miltsov, S.; Alonso, J. Characterisation of new norcyanine dyes and their application as $\mathrm{pH}$ chromoionophores in optical sensors. Dyes Pigm. 2007, 73, 383-389.

(8) Lynch, D. E.; Hamilton, D. G. Croconaine Dyes - the Lesser Known Siblings of Squaraines. Eur. J. Org. Chem. 2017, 2017, 38973911.

(9) Spence, G. T.; Hartland, G. V.; Smith, B. D. Activated photothermal heating using croconaine dyes. Chem. Sci. 2013, 4, $4240-4244$.

(10) Guha, S.; Shaw, G. K.; Mitcham, T. M.; Bouchard, R. R.; Smith, B. D. Croconaine rotaxane for acid activated photothermal heating and ratiometric photoacoustic imaging of acidic $\mathrm{pH}$. Chem. Commun. 2016, 52, 120-123.

(11) Yasui, S.; Matsuoka, M.; Kitao, T. Syntheses and some properties of infrared-absorbing croconium and related dyes. Dyes Pigm. 1989, 10, 13-22.

(12) Harmatys, K. M.; Battles, P. M.; Peck, E. M.; Spence, G. T.; Roland, F. M.; Smith, B. D. Selective photothermal inactivation of cells labeled with near-infrared croconaine dye. Chem. Commun. 2017, 53, 9906-9909.

(13) Ye, S.; Liang, Q.; Li, Z.; Xu, S.; Yao, C. A highly sensitive and selective naked-eye probe for detection of $\mathrm{Fe} 3+$ based on a 2,5-bis[3benzyl-2-methylbenzothiazole]-croconaine. Tetrahedron 2017, 73, $1350-1357$.

(14) Wang, S.; Cong, T.; Liang, Q.; Li, Z.; Xu, S. Dual colorimetric and fluorescent chemosensor of $\mathrm{Fe} 3+$ and $\mathrm{Cu} 2+$ based on 2,5-bis[ $[(4-$ carboxylic-piperidylamino)thiophenyl]-croconine. Tetrahedron 2015, $71,5478-5483$

(15) Jeppesen, A.; Nielsen, B. E.; Larsen, D.; Akselsen, O. M.; Sølling, T. I.; Brock-Nannestad, T.; Pittelkow, M.; Wang, X.; Cui, S.; Guo, K.; et al. Croconamides: a new dual hydrogen bond donating motif for anion recognition and organocatalysis. Org. Biomol. Chem. 2017, 15, 2784-2790.

(16) Li, Z.; Jin, Z. hui; Kasatani, K.; Okamoto, H. Resonant thirdorder optical nonlinearities of 1,3-bis[(3,3-dimethylindolin-2ylidene)methyl]croconine. Phys. B 2006, 382, 229-234.

(17) Punzi, A.; Capozzi, M. A. M.; Fino, V.; Carlucci, C.; Suriano, M.; Mesto, E.; Schingaro, E.; Orgiu, E.; Bonacchi, S.; Leydecker, T.; et al. Croconaines as molecular materials for organic electronics: synthesis, solid state structure and use in transistor devices. J. Mater. Chem. C 2016, 4, 3138-3142.

(18) Tang, L.; Zhang, F.; Yu, F.; Sun, W.; Song, M.; Chen, X.; Zhang, X.; Sun, X. Croconaine nanoparticles with enhanced tumor accumulation for multimodality cancer theranostics. Biomaterials 2017, 129, 28-36.

(19) Hillenkamp, F.; Peter-Katalinic, J. MALDI MS: A Practical Guide to Intrumentation, Methods and Applications, 2nd ed.; WileyBlackwell, 2013; p 477.
(20) Calvano, C. D.; Monopoli, A.; Cataldi, T.; Palmisano, F. MALDI matrices for low molecular weight compounds: an endless story? Anal. Bioanal. Chem. 2018, 410, 4015-4038.

(21) Calvano, C. D.; Ventura, G.; Cataldi, T. R. I.; Palmisano, F. Improvement of chlorophyll identification in foodstuffs by MALDI $\mathrm{ToF} / \mathrm{ToF}$ mass spectrometry using 1,5-diaminonaphthalene electron transfer secondary reaction matrix. Anal. Bioanal. Chem. 2015, 407, 6369-6379.

(22) Calvano, C. D.; Ventura, G.; Trotta, M.; Bianco, G.; Cataldi, T. R. I.; Palmisano, F. Electron-Transfer Secondary Reaction Matrices for MALDI MS Analysis of Bacteriochlorophyll a in Rhodobacter sphaeroides and Its Zinc and Copper Analogue Pigments. J. Am. Soc. Mass Spectrom. 2017, 28, 125-135.

(23) Calvano, C. D.; Ventura, G.; Palmisano, F.; Cataldi, T. R. I. 4 Chloro- $\alpha$-cyanocinnamic acid is an efficient soft matrix for cyanocobalamin detection in foodstuffs by matrix-assisted laser desorption/ionization mass spectrometry (MALDI MS). J. Mass Spectrom. 2016, 51, 841-848.

(24) Vermillion-Salsbury, R. L.; Hercules, D. M. 9-Aminoacridine as a matrix for negative mode matrix-assisted laser desorption/ ionization. Rapid Commun. Mass Spectrom. 2002, 16, 1575-1581.

(25) Wyatt, M. F.; Stein, B. K.; Brenton, A. G. Characterization of various analytes using matrix-assisted laser desorption/ionization time-of-flight mass spectrometry and 2-[(2E)-3-(4-tert- butylphenyl)2-methylprop-2-enylidene]malononitrile matrix. Anal. Chem. 2006, $78,199-206$.

(26) Zhan, C. G.; Nichols, J. A.; Dixon, D. A. Ionization potential, electron affinity, electronegativity, hardness, and electron excitation energy: Molecular properties from density functional theory orbital energies. J. Phys. Chem. A 2003, 107, 4184-4195.

(27) Johansson, T.; Mammo, W.; Svensson, M.; Andersson, M. R.; Inganäs, $\mathrm{O}$. Electrochemical bandgaps of substituted polythiophenes. J. Mater. Chem. 2003, 13, 1316-1323.

(28) Hoteling, A. J.; Nichols, W. F.; Giesen, D. J.; Lenhard, J. R.; Knochenmuss, R. Electron Transfer Reactions in Laser Desorption/ Ionization and Matrix-Assisted Laser Desorption/Ionization: Factors Influencing Matrix and Analyte Ion Intensities. Eur. J. Mass Spectrom. 2006, 12, 345-358.

(29) Knochenmuss, R. Photoionization pathways and free electrons in UV-MALDI. Anal. Chem. 2004, 76, 3179-3184.

(30) Knochenmuss, R.; Zenobi, R.; R. Knochenmuss, A.; Zenobi, R. MALDI ionization: The role of in-plume processes. Chem. Rev. 2003, 103, 441-452.

(31) Ulmer, L.; Mattay, J.; Torres-Garcia, H. G.; Luftmann, H. The use of 2-[(2E)-3-(4-Tert-Butylphenyl)-2-Methylprop-2-enylidene]malononitrile as a matrix for matrix-assisted laser desorption/ ionization mass spectrometry. Eur. J. Mass Spectrom. 2000, 6, 49-52.

(32) Maier, J. P. Photoelectron Spectroscopy ofperi-Amino Naphthalenes. Helv. Chim. Acta 1974, 57, 994-1003.

(33) Perrone, P. A.; Reuter, W. M.; Neubauer, K. R.; Bosnak, C. P.; Hall, G. A.; Grosser, Z. A. Bromine Speciation by HPLC/ICP-MS; PerkinElmer, Inc., 2005; pp 1-4.

(34) Guzowski, J. P.; Hieftje, G. M. Characterization of switched direct current gas sampling glow discharge ionization source for the time-of-flight mass spectrometer. J. Anal. At. Spectrom. 2000, 15, 2736.

(35) Ray, S. J.; Andrade, F.; Gamez, G.; McClenathan, D.; Rogers, D.; Schilling, G.; Wetzel, W.; Hieftje, G. M. Plasma-source mass spectrometry for speciation analysis: State-of-the-art. J. Chromatogr. A 2004, 1050, 3-34.

(36) Zhan, C. G.; Nichols, J. A.; Dixon, D. A. Ionization potential, electron affinity, electronegativity, hardness, and electron excitation energy: Molecular properties from density functional theory orbital energies. J. Phys. Chem. A 2003, 107, 4184-4195.

(37) Vasil'ev, Y. V.; Khvostenko, O. G.; Streletskii, A. V.; Boltalina, O. V.; Sotirios G. Kotsiris, A.; Drewello, T. Electron Transfer Reactivity in Matrix-Assisted Laser Desorption/Ionization (MALDI): Ionization Energy, Electron Affinity and Performance of the DCTB 
Matrix within the Thermochemical Framework. J. Phys. Chem. A 2006, 110, 5967-5972. 